\title{
FACTIBILIDAD DE LA PRODUCCIÓN MASIVA DE ALEVINES MACHOS DE Tilapia nilotica A TRAVÉS DE LA INVERSIÓN HORMONAL DE SEXO EN HONDURAS1
}

\author{
Bartholomew W. Green ${ }^{2}$, Luis A. López ${ }^{3}$
}

\section{COMPENDIO $^{4}$}

La demanda de alevine machos de tilapia para sembrar en estanques de engorde ha aumentado significativamente en los últimos cinco años. Nuevas tecnologías de producción son necesarias para poder abastecer la demanda existente y futura de alevines. Una de ellas es la Inversión hormonal de sexo en pescaditos de tilapia que consiste en la administración por vía oral de una hormona masculina sintética (17 a-metilo-testosterona) durante un período de 28 días comenzando poco después de haber eclosionado y antes de haberse diferenciado el tejido gonadal. El objetivo de este trabajo, que se desarrolló durante el período enero a noviembre de 1988, fue determinar la factibilidad de implementar en la Estación Experimental Acuícola El Carao, Comayagua, Honduras, la producción masiva de alevines machos de tilapia utilizando la Inversión hormonal de sexo. El proceso requiere pescaditos menores de $13 \mathrm{~mm}$ de largo que se obtienen de estanques de reproducción ( 0.05 - 0.1 ha) que han sido sembrados con reproductores de Tilapia nilotica ( 2 hembras: 1 macho). Los estanques son drenados en promedio de 18 a 20 días después de sembrados, los reproductores transferidos a piletas de concreto Y las larvas cosechadas usando una red de mano con malla de $1.6 \mathrm{~mm}$. El ciclo completo (siembra a siembra) de producción de larvas dura un promedio de $23 \mathrm{~d}$. El número promedio de larvas cosechadas variaba entre 66,500 a 99500 . Los pescaditos son sembrados $\left(4,400 / \mathrm{m}^{2}\right)$ en japas (encierro de malla de $1.6 \mathrm{~mm}$; dimensiones $2 \mathrm{~m} \mathrm{x} 2.1 \mathrm{~m} \mathrm{x} 1 \mathrm{~m}$ ó $1 \mathrm{~m}$ x $2 \mathrm{~m}$ x $1 \mathrm{~m}$, profundidad promedia de agua $=60 \mathrm{~cm}$ ) después de haber sido pasados por un excluidor con malla de $3.2 \mathrm{~mm}$. La hormona es Incorporada en alimento molido (23\% proteína) a razón de $60 \mathrm{mg} / \mathrm{kg}$ alimento. Alimento es ofrecido cuatro veces al día, siete días por semana. La duración de] tratamiento es de 28 d. El promedio de sobrevivencia de la etapa de tratamiento hormonal fue $87.6 \%$. Larvas tratadas son sembradas en estanques de pre-engorde $(0.2 \mathrm{ha})$ para crecimiento adicional a razón de 102,500/ha. Un total de $1.935,000$ pescaditos fueron cosechados de los estanques de reproducción, de los cuales se descartaron 350,000 por ser grandes para tratarse. De las 1,585,000 de pescaditos restantes 1.313,500 fueron sometidas a tratamiento hormonal; 272,000 fueron descartadas por falta de espacio en japas. Un total de 1.189,600 pescaditos completaron o se encontraron en la etapa de tratamiento. Estanques de pre-engorde han sido sembrados con un total de 661,700 alevines; el promedio de sobrevivencia de esta etapa fue $81.6 \%$. Hasta noviembre, 1988 , se produjeron 399,000 alevines de $18 \mathrm{~g}$ cada uno y de los cuales más del $97 \%$ fueron machos. El costo de producción de alevines tratados (0.15 g cada uno) fue L. 9.124,000 alevines. La tecnología ha resultado ser factible no sólo para la Estación Experimental Acuícola El Carao, sino también para piscicultores calificados.

Palabras clave adicionales: Hormona masculina sintética, tratamiento hormonal, piscicultura.

\begin{abstract}
The demand for young male fish of Tilapia nilotica for seeding in reproduction ponds has significantly Increased In the last five years. New production technologies are necessary in order to have adequate supply for existent and future demands of the young fish. One of those is the hormonal sexual inversion of the young fish of Tilapia nilotica via the oral Ingestion of a synthetic male hormone (17 a-methyl-testosterone) during a period of 28 days which starts shortly after hatching and before differentiation of genital tissue. The objective of this work which was conducted during the months of January through November, 1988 was to determine the feasibility of implementing the massive production of young males of Tilapia nilotica using the hormonal sexual Inversion process at the experimental station "Acuicola El Carao" in Comayagua, Honduras. The process requires obtaining young fish less than 13 min long from reproduction ponds $(0.05-0.1 \mathrm{ha})$ that have been seeded with Tilapia nilotica ( 2 females: 1 male). The ponds are then drained within 18 to 20 days after having been seeded, the reproducers are transferred to concrete separating tanks and the young fish are harvested by hand using a net $1.6 \mathrm{~mm}$ long. The complete cycle for young fish production lasts approximately 23 days. The average number of harvested fish varies between 66,500 to 99500 . The fish are seeded at a population of $4,400 / \mathrm{m}^{2}$ in "japas" (wire nets of $1.6 \mathrm{~mm}$, with dimensions $2 \mathrm{~m} \times 2.5 \mathrm{~m} \times \mathrm{I} \mathrm{m}$ or $1 \mathrm{~m} \times 2 \mathrm{~m} \times \mathrm{I} \mathrm{m}$, with an average water depth of $60 \mathrm{~cm}$ ) after having been passed through a $3.2 \mathrm{~mm}$ separator net. The hormone is incorporated into the ground feed (23 $\%$ protein) at a rate of $60 \mathrm{mg} \mathrm{kg}^{-1}$. The ground reed Is applied four times per day, seven days a week. The duration of the treatment described is 28 days. The average life span of the hormonal treatment was $87.6 \%$. Treated fish were seeded in prefattening ponds $(0.2 \mathrm{ha})$ for additional growth $\left(102,500 \mathrm{ha}^{-1}\right)$. A total of $1.935,000$ fish were harvested from the reproduction ponds of which 350,000 were discarded due to excess size. Of the $1.595,000$ fish, 1.313,500 were treated with the hormone. Of that $1.199,600$ fish were found to complete the treatment cycle. Of the seeded pre-fattening ponds with a total of $661,7 \mathrm{~W}$ fish an average survival rate of $81.6 \%$ was obtained. Up till November, 1988, 3,000 $18 \mathrm{~g}$ fish were produced, $97 \%$ being males. The cost of production of treating the fish $(0.15 \mathrm{~g}$ each) was L 9.12/1000. This technology has resulted in being feasible not only for the experiment station in Comayagua but should also be
\end{abstract} feasible for qualified fisheries

\footnotetext{
${ }^{1}$ Presentado en la XXXV Reunión Anual del PCCMCA, San Pedro Sula Honduras 1989

${ }^{2}$ Department of Fisheries and Allied Aquacultures, Auburn University, AL 36849-5419 U.S.A.

3 Estación Experimental Acuícola El Carao, Recursos Naturales Renovables, Comayagua, Honduras.

${ }^{4} \mathrm{El}$ abstract y compendio fueron elaborados por el Comité Editorial para mostrar el formato de presentación de los artículos.

Publicado en Agronomía Mesoamericana Vol. 1 (1990).
} 


\section{INTRODUCCIÓN}

La Estación Experimental Acuícola "El Carao", recientemente nombrado el Centro Nacional de Investigación Piscícola "El Carao", Recursos Naturales Renovables, Comayagua, Honduras, es la fuente principal de alevines de tilapia, las carpas y el tambaquí en el país. Los piscicultores hondureños se han acostumbrado a comprar alevines de 10 a 20 gramos cada uno, listos para sembrar en el estanque de engorde, así obviando la necesidad de que. él mismo los produzca. La Estación "El Carao" cuenta con aproximadamente 2.0 ha de estanques disponibles para la etapa de preengorde durante la cual el pez joven de ( \pm 1 gramo) crece al tamaño deseado. Por lo tanto, el número total de alevines de 10 a 20 gramos que puede producirse es limitado. Nuevos sistemas de producción de alevines fueron implementados en la Estación "El Carao" durante los últimos cuatro años para poder abastecer la demanda creciente. $\mathrm{Hu}$ bo aumentos significativos en la producción de alevines como resultados de investigaciones realizadas por el Programa Colaborativo de Apoyo a la Investigación (CRSP) en materia de acuicultura, los cuales le permitieron a la estación atender los pedidos, pero en forma demorada. Estos trabajos se enfocaron en la producción de] híbrido tilapia nilotica $x$ T. Hornorum. El híbrido fue producido debido al alto porcentaje de machos (casi $100 \%$ ) obtenidos, sin embargo, su producción está sujeta a problemas potenciales y serios al comprometer la pureza de los progenitores. Es difícil mantener la pureza de los reproductores, aun bajo las mejores circunstancias, y una vez contaminadas, es poca la justificación para producir el híbrido.

La producción de alevines machos de tilapia por la inversión hormonal de sexo es una tecnología relativamente nueva, pero ya probada. Esta tecnología consiste en la administración oral de una hormona masculina sintética durante un período de 28 días, comenzando poco después de eclosionarse y antes de diferenciarse el tejido gonodal. Al completarse el tratamiento todos o casi todos los alevines son machos funcionales. La producción masiva de alevines machos por inversión hormonal se ha demostrado en Israel (Rothbard et al, 1983), en donde las larvas fueron tratadas en agua limpia y, luego en Ecuador (Popma, 1987), en donde las larvas fueron tratadas en estanques fértiles. La presencia de productividad natural no impide la inversión de sexo. El objetivo principal de este trabajo fue determinar la factibilidad de la producción masiva de alevines machos de T. nilotica a través de la inversión hormonal de sexo en la Estación Experimental Acuícola "El Carao".

\section{MATERIALES Y MÉTODOS}

Este estudio se llevó a cabo en la Estación Experimental Acuícola El Carao", Recursos Naturales Renovables, Comayagua, Honduras, durante el período 21 de enero al 14 de noviembre de 1988. La producción de larvas de T. nilotica se realizó en estanques de 0.1 - 60.05 -ha, cada uno equipado con una pila de cosecha de concreto cuya área fue $9 \mathrm{~m}^{2}$ Una malla (luz de malla de 19.0 - $25.4 \mathrm{~mm}$ cuadrados) fue colocada encima de la pila de cosecha. Se llenaron los estanques con agua de riego filtrado por mafia "saran" y se sembraron los reproductores (Cuadro 1). El estanque fue fertilizado una vez con gallinaza $(2200 \mathrm{~kg} / \mathrm{ha})$ después de llenarse. Los reproductores fueron alimentados con alimento peletizado ( $23 \%$ proteína al $3 \%$ de la biomasa de peces por día) seis días por semana. En la cosecha que se realizó de 14 a 26 días después de sembrados, el nivel de agua del estanque fue bajado al nivel con la pila de cosecha. Tela metálica prevenía la fuga de larvas por el tubo de drenaje. Los reproductores fueron sacados de la pila en un solo lote al levantar la malla previamente colocada. Los reproductores fueron mantenidos en pilas de concreto hasta resembrarlos. Las larvas fueron cosechadas utilizando una red de mano $(1 \mathrm{~m}$ x $0.5 \mathrm{~m}$ con red de $1.6 \mathrm{~mm}$ luz de malla) y puestas en una japa (1,6 mm luz de malla) colocada en un tanque de transporte conteniendo agua limpia. Se suspendió la cosecha cuando pocas larvas fueron capturadas (probablemente menos de 500). Larvas atrapadas en la malla del tubo de drenaje o no cosechadas no fueron enumeradas. Se volvían a llenar los estanques 1 a 3 días después de drenados; el estanque vacío fue secado y/o los pozos fueron envenenados con cloro.

Las larvas cosechadas del estanque de reproducción fueron sembradas en japas para tratamiento con hormona; las japas fueron colocadas en un estanque de 0.2-ha (Cuadro 2). Generalmente las larvas fueron pasadas por un excluidor, cuya luz de malla fue $3.2 \mathrm{~mm}$, antes de sembrarse en las japas; larvas retenidas por el excluidor eran predominantemente mayores de $14 \mathrm{~mm}$ de largo, un tamaño considerado muy grande para tratar; el tamaño ideal es de 7 a $13 \mathrm{~mm}$ de largo. El estanque fue fertilizado periódicamente con gallinaza $(1,000 \mathrm{~kg} / \mathrm{ha})$ para mantener la florescencia de algas. Hubo dos tamaños de japa, dependiendo del número de larvas a tratarse: Una grande ( $2 \mathrm{~m} \times 2.5 \mathrm{~m} \mathrm{x} 1 \mathrm{~m} ; 5 \mathrm{~m}^{2}$ área) o una pequeña (1 $\mathrm{m} \times 2 \mathrm{~m} \times 1 \mathrm{~m} ; 2 \mathrm{~m}^{2}$ área). La profundidad del agua en la japa promediaba $0.6 \mathrm{~m}$. Un anillo flotante fue puesto en cada japa para contener el alimento tratado ofrecido a los peces; un pedazo de plástico fue sumergido debajo del anillo pan retener cualquier alimento que se hundió. Cada japa fue sembrada con larvas que atravesaron el excluidor a razón de 3000-5000 larvas $/ \mathrm{m}^{2}$. El número de larvas sembrado fue determinado por el método de comparación visual: una muestra de 1000-2000 larvas fue contada a un balde blanco conteniendo agua limpia; 
larvas fueron agregadas a otro balde con la misma cantidad de agua hasta que las densidades de larvas se parecieran. Una muestra de 250-500 larvas fue llevada al laboratorio para medirse individualmente y pesarse en masa. Después de la cosecha las larvas tratadas fueron sembradas en estanques de pre-engorde para crecer a un tamaño sexable.

La hormona fue incorporada en un alimento peletizado comercial de $23 \%$ proteína. El alimento peletizado fue molido usando un molino de martillo con saranda de aperturas de $9.5 \mathrm{~mm}$. El alimento molido fue pasado por una saranda de aperturas de 560 micrones para eliminar las partículas grandes. Hormona masculina sintética (17 a-metilo-testosterona) fue incorporada en el alimento sarandeado, a razón de $60 \mathrm{mg} / \mathrm{kg}$ alimento. La hormona fue disuelta primero en alcohol etílico (95\% conteniendo glicerina $0.5 \% \mathrm{v} / \mathrm{v}$ ), utilizando 0.51 de alcohol por $\mathrm{kg}$ de alimento. La solución de hormona/ alcohol entonces fue mezclada con el alimento. Luego el alimento fue secado en un horno a 60T. Alimento tratado seco fue guardado en refrigeración hasta usarse.

Una vez completado el tratamiento, los peces tratados fueron sembrados en estanques de pre-engorde de 0.2-ha cada una, a razón de 102,500/ha para crecer a un tamaño sexable.

Cuadro 1. Descripción del manejo de los estanques para la producción de larvas de Tilapia nilotica a someterse a la inversión hormonal de sexo en la Estación Experimental Acuícola "El Cano", Comayagua, Honduras. Datos son de cosechas completas de estanques durante el período enero a noviembre, 1988

\begin{tabular}{|c|c|c|}
\hline Descripción & Promedio & Rango \\
\hline \multicolumn{3}{|l|}{ ESTANQUE DE O.1-ha } \\
\hline Número hembras sembradas & 245 & $150-400$ \\
\hline Biomasa hembras sembradas (kg) & $63.57 \mathrm{P}$ & $32.829-142.344$ \\
\hline Número machos sembrados & 130 & $72-201$ \\
\hline Biomasa machos sembrados $(\mathrm{kg})$ & 47.727 & 24.608-91.995 \\
\hline Relación hembra:macho & 1.95 & $1.62-2.11$ \\
\hline Duración siembra-cosecha (d) & 20 & $16-26$ \\
\hline Duración siembra-resiembra (d) & 23 & $19-34$ \\
\hline \multicolumn{3}{|l|}{$\begin{array}{l}\text { Número de larvas cosechadas } \\
\text { (x 1000) }\end{array}$} \\
\hline$\leq 3 \mathrm{~mm}$ largo & 59.8 & $22-134.1$ \\
\hline$\geq 214 \mathrm{~mm}$ largo & 39.7 & 0.0180 .8 \\
\hline Total & 99.5 & $16.0-183.0$ \\
\hline \multicolumn{3}{|l|}{ ESTANQUE DE 0.05-ha } \\
\hline Número hembras sembradas & 209 & $115-250$ \\
\hline Biomasa hembras sembradas (kg) & 40.131 & $10.518-60.380$ \\
\hline Número machos sembrados & 96 & $69-117$ \\
\hline Biomasa machos sembrados $(\mathrm{kg})$ & 26.097 & $18.711-31.483$ \\
\hline Relación hembra:macho & 2.23 & $1.50-2.62$ \\
\hline Duración siembra-cosecha (d) & 18 & $14-22$ \\
\hline Duración siembra-resiembra (d) & 23 & $16-31$ \\
\hline \multicolumn{3}{|l|}{ Número de larvas cosechadas (x 1000) } \\
\hline$\leq 13 \mathrm{~mm}$ largo & 59.5 & 5.6-110.9 \\
\hline$\geq 14 \mathrm{~mm}$ largo & 7.0 & $0.0-43.0$ \\
\hline Total & 66.5 & $83-110.9$ \\
\hline
\end{tabular}

* Dimensiones: $2 \mathrm{~m}$ x $2.5 \mathrm{~m}$ x $1 \mathrm{~m}$

**Dimensiones: $1 \mathrm{~m} \mathrm{x} 2 \mathrm{~m}$ x $1 \mathrm{~m}$
El estanque fue llenado aproximadamente una semana antes de sembrarse y fertilizado con gallinaza (2000 kg/ha). Gallinaza fue aplicada semanalmente durante el período de preengorde a razón de $1000 \mathrm{~kg} / \mathrm{ha}$. Los alevines fueron alimentados cinco días por semana a razón de $4.5 \mathrm{~kg}$ alimento (23\% proteína)/día. La duración de la etapa de pre-engorde fue de 45-60 días. En 16 de 21 cosechas, 5000 alevines por estanque fueron sexados para determinar la eficacia del tratamiento hormonal. En seis ocasiones, lotes de 100 peces por lote tratado fueron examinados microscópicamente para determinar el sexo; los resultados eran $\pm 0.5 \%$ de los obtenidos con el sexado manual.

Se realizó un análisis económico sobre producir anualmente 750,000 larvas tratadas de $0.15 \mathrm{~g}$ cada uno, listos para sembrar en el estanque de pre-engorde. El sistema requería dos estanques de 0.05 -ha: Uno para reproducción y el otro para tratamiento hormonal. La hormona y cierto equipo tienen que ser importados; sus costos reflejan transporte y $100 \%$ impuesto de introducción. El costo de la etapa de pre-engorde no se incluyó.

Cuadro 2. Resumen de resultados de la etapa de tratamiento hormonal en la producción de alevines machos de Tilapia hormonal en la Estación Experimental Acuícola "El Carao", Comayagua. Hormona masculina sintética (17 a-metilo-testosterona) fue incorporada en alimento molido a razón de $60 \mathrm{mg} / \mathrm{kg}$. Datos son cosechas totales de Japas entre 16 de febrero y 14 de noviembre 1988

\begin{tabular}{|c|c|c|}
\hline Descripción & Promedio & Rango \\
\hline \multicolumn{3}{|l|}{$\begin{array}{l}\text { NUMERO DE LARVAS SEMBRADAS } \\
\text { (x 1000) }\end{array}$} \\
\hline Total japa* pequeña & 8.7 & $6.0-10.0$ \\
\hline Total japa** grande & 21.5 & $10.0-26.0$ \\
\hline Por $\mathrm{m}^{2} \mathrm{de}$ japa pequeña & 4.4 & 3.03 .0 \\
\hline Por $\mathrm{m}^{2}$ de japa grande & 4.3 & 2.03 .2 \\
\hline \multicolumn{3}{|l|}{ Peso promedio inicial (g/larva) todas } \\
\hline \multicolumn{3}{|c|}{ Tamaño inicial de larvas $(\%)$ todas larvas } \\
\hline$\leq 13 \mathrm{~mm}$ largo & 89.3 & 40.6400 .0 \\
\hline 14-15 mm largo & 6.9 & $0.0-29.7$ \\
\hline$\geq 16 \mathrm{~mm}$ largo & 3.9 & $0.0-31.2$ \\
\hline \multicolumn{3}{|l|}{ Número de larvas cosechadas (x 1000) } \\
\hline Total jupa pequeña & 7.0 & $3.4-12.5$ \\
\hline Total japa grande & 19.1 & $0.0-32.8$ \\
\hline Por $\mathrm{m}^{2}$ japa pequeña & 3.5 & $13-6.2$ \\
\hline Por $\mathrm{m}^{2}$ japa grande & 3.8 & 0.046 \\
\hline Sobrevivencia $(\%)$ todas larvas & 88.8 & 0.0153 .5 \\
\hline Peso prom. final (g/1arva) todas larvas & 0.144 & $0.069-0.751$ \\
\hline \multicolumn{3}{|l|}{ Tamaño final de larvas (\%) todas larvas } \\
\hline$\leq 13 \mathrm{~mm}$ largo & 2.0 & $0.0-35.3$ \\
\hline$\geq 14 \mathrm{~mm}$ largo & 98.0 & $643-100.0$ \\
\hline Duración del tratarniento (d) todas larvas & s 28 & $26-28$ \\
\hline \multicolumn{3}{|l|}{ Alimento tratado usado todas larvas } \\
\hline Por japa $(\mathrm{kg})$ & 3.774 & $1.680-5.719$ \\
\hline $\mathrm{kg} / 1000$ larvas cosechadas & 0.213 & $0.096-0.752$ \\
\hline \multicolumn{3}{|l|}{ Machos fenotípicos producidos (\%) } \\
\hline todas larvas & 97.8 & $903-99.5$ \\
\hline
\end{tabular}

* Dimensiones: $2 \mathrm{~m}$ x $2.5 \mathrm{~m} \mathrm{x} 1 \mathrm{~m}$

** Dimensiones: $1 \mathrm{~m}$ x $2 \mathrm{~m}$ x $1 \mathrm{~m}$ 


\section{RESULTADOS Y DISCUSIÓN}

Los datos de producción de larvas en el estanque de reproducción son resumidos en el Cuadro 1. Larvas mayores de $13 \mathrm{~mm}$ de largo son consideradas como muy grandes para someter a tratamiento hormonal, ya que el tejido gonadal hubiera comenzado a diferenciarse. Durante el transcurso del año hubo aumento en la temperatura del agua y por lo tanto la duración de esta etapa disminuyó de un promedio de 20 a 18 días. Como resultado de esta reducción, hubo menos producción de larvas mayores de $13 \mathrm{~mm}$ de largo, lo cual significa una utilización más eficiente del estanque de reproducción. La producción total de larvas parecía mayor en el estanque de reproducción más grande, sin embargo, la producción de larvas menores de $13 \mathrm{~mm}$ de largo fue similar en los dos tamaños de estanque.

Un promedio de $88,5 \%$ de las larvas sembradas completaron la etapa de tratamiento hormonal (Cuadro 2). La mayoría de las larvas eran mayores de $13 \mathrm{~mm}$ de largo al cosecharlas. Hasta la fecha, el tratamiento hormonal ha resultado en un promedio de $97.8 \%$ alevines machos. Es posible que el $2 \%$ de peces menores de 13 $\mathrm{mm}$ largo en la cosecha tuvieran un porcentaje significativo de hembras (peces no invertidos) (Popma, 1987). Hasta la fecha, se ha usado $137.835 \mathrm{~kg}$ tratados en producir más de 725,000 larvas tratadas.

El análisis económico de la producción de alevines (0. $15 \mathrm{~g}$ cada una) machos de Tilapia nilotica por inversión hormonal del sexo indicó que el costo total por cada 1000 alevines fue de L. 9.12 (\$1 U.S.= 2 Lempiras) (Cuadro 3). La mano de obra comprendía 528.4 hombre-horas para la etapa de producción de larvas y 1834.6 hombre-horas para la etapa de tratamiento hormonal en japas.

Un total de 1.935,000 larvas fueron cosechadas de los estanques de reproducción, de los cuales se descartaron 350,000 por ser demasiado grandes para tratar. De las $1.585,000$ de larvas restantes $1.313,500$ fueron sometidas a tratamiento hormonal; 272,000 fueron descartadas por falta de espacio en japas. Un total de 1. 189,600 larvas completaron o se encontraron en la etapa de tratamiento. Estanques de pre-engorde han sido sembrados con un total de 661,700 alevines; el promedio de sobrevivencia de esta etapa fue $81.6 \%$. Hasta noviembre 1988 , se produjeron 399,000 alevines de $18 \mathrm{~g}$ cada uno y de los cuales más del $97 \%$ fueron machos. El costo de producción de alevines tratados $(0.15 \mathrm{~g}$ cada uno) fue $\mathrm{L}$. 9.12/ 1,000 alevines. La tecnología ha resultado ser factible no sólo para la Estación Experimental Acuícola El Carao, sino también para piscicultores calificados.

\section{RECONOCIMIENTO}

Agradecemos la colaboración de Miguel Zelaya. Este trabajo fue realizado como parte del Proyecto de
Cuadro 3. Análisis económico de la producción anual de 750,000 alevines machos ( $>97 \%$ machos) de Tilapia nilotica de $0.15 \mathrm{~g}$ cada uno por inversión hormonal de sexo. Se necesitan 2 estanques de 0.05-ha: Uno como estanque de reproducción y uno para tratamiento hormonal. El ciclo de reproducción promedia 25 días de siembra a resiembra. Trece ciclos de reproducción por año son posibles. Un promedio de 67,000 larvas/cosecha son obtenidas del estanque de reproducción. Un promedio de $87.57 \%$ de las larvas sobreviven los 28 días de tratamiento con hormona. Los costos son de noviembre, 1988 (2 lempiras = \$1 U.S.).

\begin{tabular}{|c|c|}
\hline Descripción & $\begin{array}{c}\text { Costo } \\
\text { (Lempiras) }\end{array}$ \\
\hline \multicolumn{2}{|l|}{ COSTOS VARIABLES } \\
\hline \multicolumn{2}{|l|}{ Alimento } \\
\hline Ración comercial, 811 kg @ L. 0.80/kg & 648.80 \\
\hline $\begin{array}{l}\text { Ración tratada con hormona, } 162 \text { kg@ L. 4.23/kg } \\
\text { Agua de riego }\end{array}$ & 686.10 \\
\hline $\begin{array}{l}\text { Estanque de reproducción, } 0.825 \text { ha-m @ 1- 25.00/ha-m } \\
\text { Estanque de tratamiento hormonal, } 0.23 \text { ha-m @ }\end{array}$ & 20.64 \\
\hline $\begin{array}{l}\text { L. } 25.001 \text { ha-in } \\
\text { Desinfectante }\end{array}$ & 5.75 \\
\hline $\begin{array}{l}\text { Desinfectante } \\
\text { 11TH chloro, } 7.8 @ \text { L 10.50/kg } \\
\text { Transporte }\end{array}$ & 81.901 \\
\hline $\begin{array}{l}\text { Alimento, } 22 \text { sacos (d L. 1.001saco, } \\
\text { Mano de obra }\end{array}$ & 22.00 \\
\hline $\begin{array}{l}\text { Producción de larvas, } 202 \text { hombre-horas @ L.0.875/ } \\
\text { hom-h }\end{array}$ & 176.75 \\
\hline \multicolumn{2}{|l|}{ Tratamiento hormonal, 702 hombre-horas @ L.0.875/ } \\
\hline $\begin{array}{l}\text { Gerencia, prorrateado,15\% @ L. 13,2001afic, } \\
\text { Vigilante, prorrateado,4\% @ L. 2,5201año }\end{array}$ & $\begin{array}{r}1980.00 \\
100.80\end{array}$ \\
\hline Subtotal Costos Variables & 4336.99 \\
\hline \multicolumn{2}{|l|}{$\begin{array}{l}\text { Intereses sobre capital variable, } 9 \% \text { por año, calculado } \\
\text { diariamente }\end{array}$} \\
\hline TOTAL COSTOS VARIABLES & 4745.53 \\
\hline \multicolumn{2}{|l|}{ COSTOS FIJOS } \\
\hline \multicolumn{2}{|l|}{ Depreciación } \\
\hline Japas (vida 3 años) & 314.67 \\
\hline Malla, pila de cosecha (vida 3 años) & 190.00 \\
\hline \multicolumn{2}{|l|}{ Redes de Mano } \\
\hline Malla (vida 2 años) & 49.00 \\
\hline Manga (vida 5 años; $50 \%$ valor salvamento) & 10.50 \\
\hline Azafata para secar alimento (vida 5 años) & 12.00 \\
\hline Excluidor (vida 2 años) & 20.00 \\
\hline Baldes (vida 1 año) & 50.00 \\
\hline Pila de concreto (vida 5 años; $25 \%$ valor salvamento) & 150.00 \\
\hline Muelle (vida 2 años) & 85.00 \\
\hline \multicolumn{2}{|l|}{ Mantenimiento } \\
\hline Estanques, 0.1 -ha@ 240.00/ha/año & 24,00 \\
\hline Muelle, $12 \%$ costo total/año & 20.40 \\
\hline \multicolumn{2}{|l|}{ Reemplazo de reproductores ( $20 \%$ población/año, } \\
\hline Impuesto ejidal & 1.50 \\
\hline \multicolumn{2}{|l|}{ Interés sobre capital fijo, $9 \%$ por año, calculado diariamente } \\
\hline Construcción de estanques (L. 1,734.00) & 163.28 \\
\hline Muelle (L. 170.00) & 16.01 \\
\hline Equipo (L. 1,867.00) & 175.87 \\
\hline Pila de concreto (L. 1,000.00) & 94.20 \\
\hline Reproductores (L. 248.25) & 23.38 \\
\hline Cobro por tierra, 0.1 6-ha para arroz & 641.45 \\
\hline TOTAL COSTOS FIJOS & 2090.91 \\
\hline TOTAL COSTOS & 6836.44 \\
\hline \multicolumn{2}{|l|}{ COSTO TOTAL POR 1000 ALEVINES (0.15 g cada uno) } \\
\hline INVERTIDOS & 9.12 \\
\hline
\end{tabular}


Acuicultura de Agua Dulce (Contrato USAID 522-0168-C-00-8010-00).

\section{BIBLIOGRAFIA}

POPMA, T. J., 19V. Freshwater fish culture development project/ESPOL, Guayaquil, Ecuador: Final Technical Report. Auburn University, AL 36849 USA. 75 p.
ROTHBARD. S.; SOLNIK. E.; SHABBATH, S.; AMADO, R., GRABIE, 1. 1983. The technology of mass production of hormonally sex inversed all-male tilapias. $I n \mathrm{~L}$. Fishelson and Z Yaron. (ed.). International Symposium on Tilapia in Aquaculture. Tel Aviv University, Tel Aviv, Israel. p. 425434. 